\title{
DDS
}

\section{IDS 2021:}

\section{Qualität statt Quantität}

AUTORIN: BIRGIT JARETZ

Gut war's! So lautet das übereinstimmende Fazit der Besucher und Aussteller der 39. IDS. Auch die Veranstalter, der Verband der Deutschen Dental-Industrie e.V. (VDDI), die GFDI Gesellschaft zur Förderung der Dental-Industrie mbH und die Koelnmesse GmbH, bilanzierten einen zurückgekehrten Optimismus. Dieser positive Eindruck ist umso erfreulicher, als dass die außergewöhnlichen Umstände und zahlreiche Absagen einiger Big-Player der DentalBranche das Gegenteil vermuten ließen. Stattdessen hat die IDS 2021 für Mark Stephen Pace, den Vorstandsvorsitzenden des VDDI, die erhofften Impulse in der Post-Corona-Zeit gesetzt und signalisiert: Die deutsche Dental-Industrie geht voran.

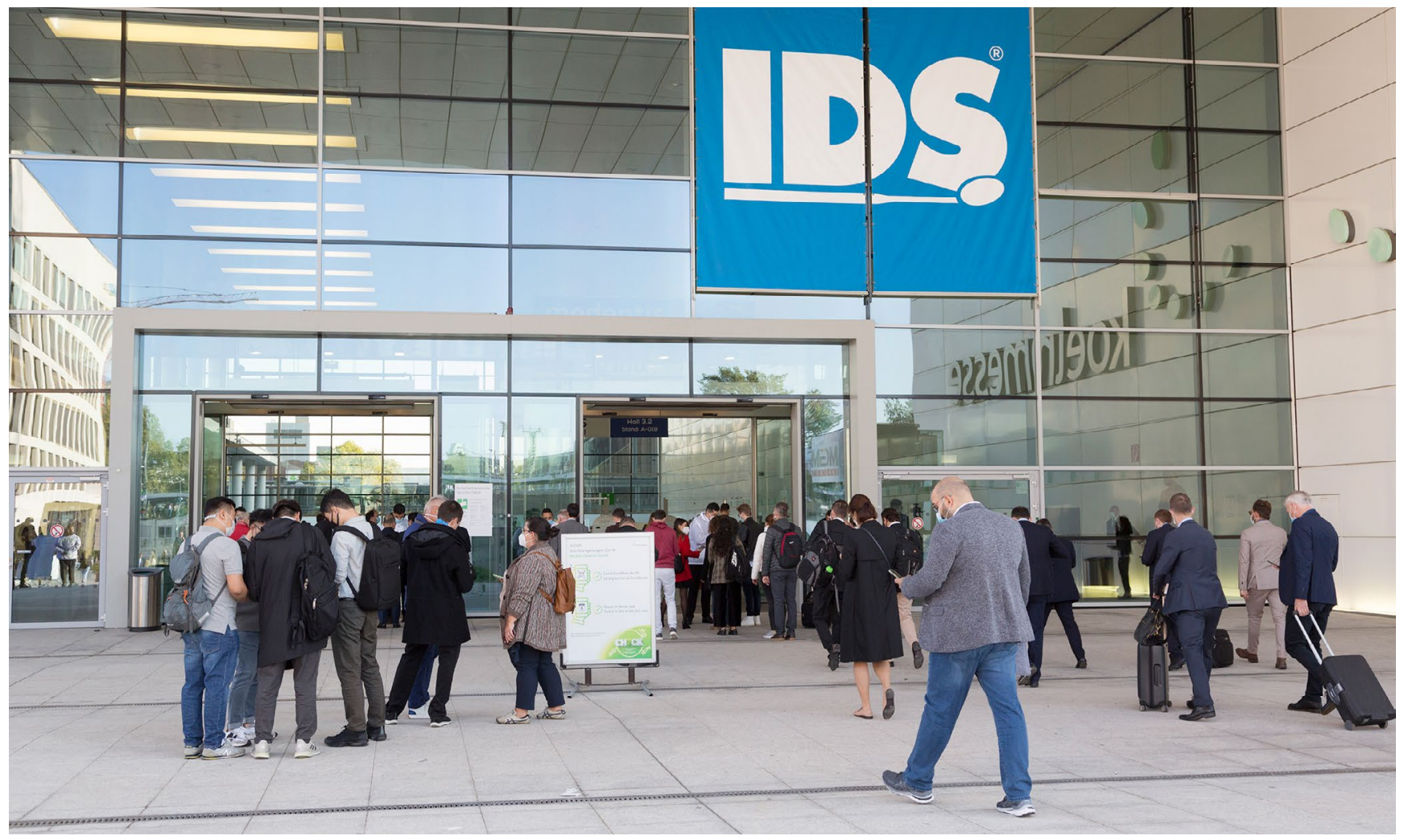

Zugegeben - ein wenig gespenstisch war das Gefühl schon beim Wandeln durch mehr oder weniger leere Hallen. Kein Wunder, denn in diesem Jahr betrug die Zahl der Aussteller mit 830 nur etwas mehr als ein Drittel der Aussteller von 2019 (2327). Auch die Besucherzahl lag mit 23.000 deutlich unter den vorgelegten 160.000 der letzten IDS.

Die Herkunft der Fachbesucher aus 114 Ländern und ihr Auslandsanteil von 57 Prozent unterstreicht dagegen die Bedeutung der IDS als Weltleitmesse der Dental-Industrie. Allen Unkenrufen zum Trotz war die schon fast totgesagte Messe dann doch ein Erfolg und hat der internationalen Dental-
Familie laut Mark Stephen Pace den Optimismus zurückgebracht. Schließlich ist nicht die Quantität, sondern die Entscheidungskompetenz der Besucher ausschlaggebend für den Erfolg einer Messe. Die war in diesem Jahr national wie international auf einem extrem hohen Niveau.

Laut einer Besucherbefragung waren nahezu 85 Prozent der diesjährigen IDS-Besucher an der Beschaffungsentscheidung beteiligt, 33 Prozent sogar auschlaggebend. Gut zwei Drittel waren mit dem Ausstellungsangebot sehr zufrieden und 85 Prozent würden einem guten Geschäftsfreund den Besuch auf der IDS empfehlen. 


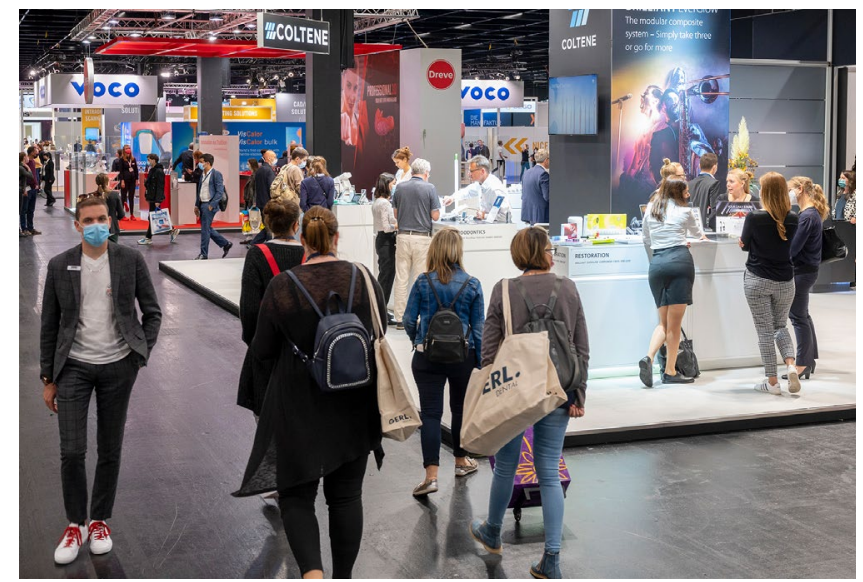

ENTSPANNTE ATMOSPHÄRE IN DEN GÄNGEN UND AN DEN STÄNDEN

\section{DAS GEHEIMNIS DES ERFOLGS: QUALITATIVE GESPRÄCHE IN ENTSPANNTER ATMOSPHÄRE}

Was steckt also hinter dem Erfolg der abgespeckten IDS-Variante? Das Zauberwort lautet Entschleunigung. Auch wenn manch einer den Austausch mit fehlenden Industriepartnern oder die Show-Acts an den Ständen vermisst hat, so waren sich Aussteller wie Besucher einig: Die Gespräche waren qualitativ hochwertiger und intensiver, schlichtweg weil beide Gesprächspartner mehr Zeit hatten und sich auf ihr Gegenüber konzentrieren konnten. Der Trend zur Entschleunigung hat sich auch bei dem zuletzt ungebremsten Wachstum der IDS durchgesetzt. So könnte die Corona-Pandemie einen Wendepunkt in dem inflationären Expansionsdrang der IDS markieren und eine zukünftige Konzentration auf das Wesentliche einleiten. Neu war in diesem Jahr das der Pandemie geschuldete hybride Format. Mit einer Beteiligung von 77 Ausstellern aus 16 Ländern und 88 täglichen Beiträgen kann auch die digitale Plattform IDSconnect als Erfolg gewertet werden. Einige Aussteller haben sich sowohl online als auch vor Ort präsentiert.

\section{ZUKUNFTSFELD KÜNSTLICHE INTELLIGENZ}

So lud zum Beispiel der Medizintechnikhersteller Dürr Dental eine Woche vor der IDS zu einer Online-Pressekonferenz zum 80. Firmenjubiläum mit kulinarischer Weinreise ein. Das schwäbische Familienunternehmen konnte so den Teilnehmern die zahlreichen Neuheiten in den Bereichen Hygiene, Praxisversorgung und Bildgebung bereits vorab in entspannter Atmosphäre zu Hause vorstellen. Während der Messe konnten sich die Besucher vor Ort von den Vorzügen des weltweit ersten Zwei-Slot-Scanners mit RFID Technologie VistaScan Ultra View, den neuen Saugsystemen Tyscor VS sowie dem Dampf-Sterilisator Hygoclav überzeugen. Nicht nur Dürr Dental legte den Schwerpunkt auf den Bereich Imaging mit Fokus auf künstlicher Intelligenz (KI). Bei weiteren KI-Projekten steht die Auswertung von Röntgenbildern im Mittelpunkt. Die bestehende Software könnte dafür genutzt werden und mithilfe zusätzlicher Bilddaten oder auch klinischer Informationen eine hilfreiche Unterstützung für die Therapie-Entscheidung sein.

\section{HYGIENE UND INFEKTIONSKONTROLLE WICHTIGER DENN JE}

Die Corona-Pandemie hat die ohnehin in der Zahnarztpraxis essenziellen Maßnahmen zur Hygiene und Infektionskontrolle in den Fokus gerückt. Entsprechend wurden auf der IDS unter anderem leistungsfähige Saugsysteme zur AerosolReduktion vorgestellt. Ganz neu sind Kopfhörer mit einer daran befestigten Absaugkanüle von SycoTec. Die potenziell keimbelastete Aerosolwolke kann damit direkt am Austritt des Patientenmunds abgesaugt werden und die Aerosolbildung im Praxisraum um bis zu 99,9 Prozent verringern. Laut einer auf der IDS vorgestellten Studie der Université Claude Bernhard, Lyon, kann die Mundspülung Perio plus Regenerate von Curaprox das Risiko der Übertragung von SARSCoV-2 verringern, indem sie die Virenlast im Mund nach einer einzigen Spülung um 71 Prozent vermindert.

\section{NEUARTIGES SCHIENENKONZEPT UND AUTOMATISIERTES HER- STELLUNGSVERFAHREN BEI ALIGNERN}

Neue Entwicklungen konnten die IDS-Besucher bei der Aligner-Therapie entdecken: Dentaurum stellte ein neuartiges Zwei-Schienen-Konzept vor, bei dem pro Behandlungsschritt zwei Folienstärken verwendet werden, um die Kräfte optimal auf die Zähne zu übertragen. Weiche und harte Aligner werden wöchentlich gewechselt, was zu einer schonenden Zahn-

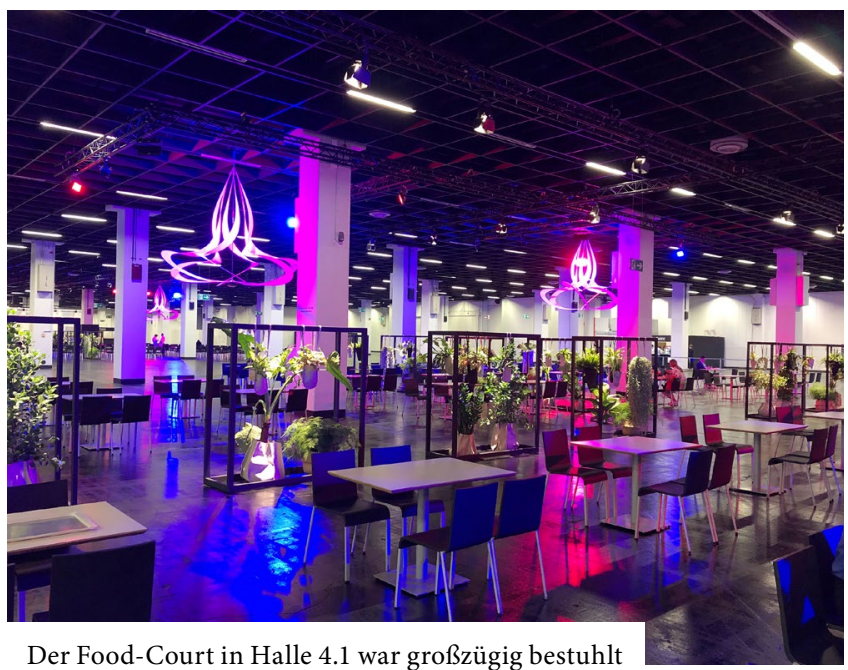
und folgte einem hygienischen Leitsystem. 


\section{NACHGEFRAGT: WIE WAR DIE IDS?}

\section{Der persönliche Austausch vor Ort ist durch nichts zu ersetzen}

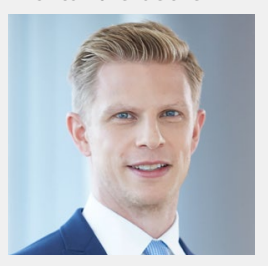

„Die Atmosphäre auf der diesjährigen IDS war aufgrund weniger Aussteller, Besucher und großflächigen Abständen zwischen den Ständen zweifelsfrei eine andere. Bei vielen Ausstellern und Besuchern war jedoch die Freude spürbar, mit der IDS endlich wieder eine Veranstaltung zu haben, um mit Kunden und Partnern persönlich in Kontakt treten zu können.

Mit der Resonanz an unserem Kreussler Pharma Stand waren wir sehr zufrieden. Die Nachfrage nach unseren Produkten sowie viele interessante und gute Gespräche am Stand haben uns in der Entscheidung für eine Teilnahme als Aussteller bestätigt. Positiv überrascht hat uns zudem der Anteil ausländischer Besucher, die sich für unsere Produkte interessiert haben."

Stefan Schneider, Kreussler Pharma

\section{Weniger Quantität - deutlich mehr} Qualität

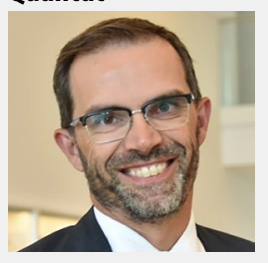

${ }_{\text {„Für VOCO war diese }}$ IDS ein Erfolg. Unsere Messestände waren über alle Tage gut frequentiert. Zwar haben wir den Austausch mit fehlenden Industriepartnern vermisst, die Atmosphäre war aber gefühlt etwas chilliger als die letzten Messen, und der weniger starke Zeitdruck ist von allen gut genutzt worden. Die Kunden, die den Weg nach Köln gefunden haben - nationale wie auch internationale - hatten mehr Zeit für deutlich intensivere Beratungsgespräche. Unterm Strich: weniger Quantität - deutlich mehr Qualität. Unsere Kunden und Partner haben sich besonders über den persönlichen Austausch gefreut, denn der hat vielen in den letzten 18 Monaten gefehlt. Auch die Rückmeldungen zu den Inhalten der IDSconnect waren durchweg positiv - hier hätten wir uns aber über höhere Teilnehmerzahlen gefreut. Aus unserer Sicht hat die IDS den Anspruch der Weltleitmesse dieses Jahr weiter ausgebaut und wird dies auch 2023 tun. Wir beginnen schon mit den Vorbereitungen für 2023 und erwarten auch hier wieder erfolgreiche Tage in Köln - hoffentlich mit wieder mehr Besuchern aus der ganzen Welt!"

Dr. Axel Bernecker, Voco

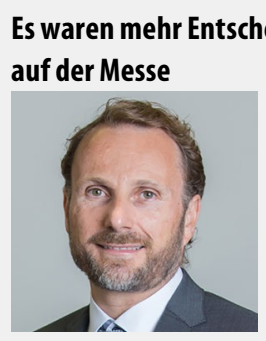

\section{Es waren mehr Entscheidungsträger auf der Messe}

hochkarätig besetzten Podiumtalks den Zuschauern zusätzlich spannende Themen bieten konnten. Durch die geringeren Aussteller- und Besucherzahlen wirkte die IDS wesentlich entspannter. Aussteller und Besucher hatten mehr Zeit für die Gespräche, und es wurden bereits am Stand Entscheidungen getroffen, weil in diesem Jahr deutlich mehr Entscheidungsträger auf der
Messe waren als üblich. Die Qualität der Kundengespräche war durch die entspannte Atmosphäre sehr gut. Das neue hybride Format der Messe hat sich als erfolgreich erwiesen, und daher denken wir, dass es bei der nächsten IDS beibehalten wird."

\section{Reinhard Larch, XO CARE}

\section{Wir haben sehr positives Feedback zu unserem Online-Portal erhalten}

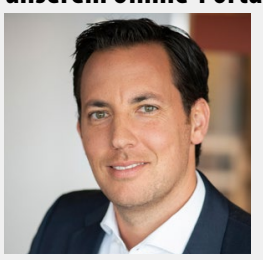
„Die IDS ist die größte Dentalfachmesse der Welt und eine der wichtigsten Veranstaltungen für die gesamte Branche. In diesem Jahr waren wir gemeinsam mit exocad mit der bisher größten Ausstellungsfläche $\left(600 \mathrm{~m}^{2}\right)$ auf der Messe vertreten, auf der wir zum ersten Mal die Align Digital Platform einschließlich der CAD/CAM-Lösungen von exocad vorgestellt haben. Es gab zwar weniger Besucher als sonst, aber wir konnten viele sehr gute Kundengespräche führen. Darüber hinaus haben wir mit unserem Online-Portal weltweit interessierten Zahnärzten die Möglichkeit gegeben, die Messe aus der Ferne zu erleben und Zugang zu all unseren Live-Präsentationen und Q\&A Sessions zu erhalten und eine sehr positive Resonanz erfahren. Wir können die Zukunft nicht vorhersagen, aber wir können auf eine fesselnde und positive IDS-Erfahrung zurückblicken. Wir freuen uns schon jetzt auf die nächste IDS, die Jubiläumsausgabe im Jahr 2023."

Daniel Echouafni, Align Technology umstellung führt. Nach vorausgehender digitaler Behandlungsplanung erhält die Praxis sowohl die Aligner als auch alle Patienten-Informationen als Komplettpaket.

Für die automatisierte Fertigung der unsichtbaren Zahnschienen im Labor bietet imes-icore ein neuartiges Produktionssystem auf Basis der international bewährten CORiTEC 350i CAD/CAM-Maschine an (Kapazität: bis zu 1000 Stück pro Tag). Der Vorteil ist die Nachverfolgung jeder Arbeit im Produktionsprozess, denn üblicherweise war hierfür ein Modell (zum Beispiel mit aufgedrucktem Code) erforderlich, während nun der Aligner direkt im Maschinensystem mit einem Laser eindeutig und permanent markiert wird.
Align Technology präsentierte sein digitales Portfolio gemeinsam mit exocad auf seiner bisher größten IDS-Ausstellungsfläche. Der interaktive Stand trug dem Hybrid-Konzept des Entwicklers des Invisalign Systems Rechnung: Die Besucher vor Ort konnten in den Stationen Connect, Scan, Plan, Treat, Monitor und Restorative Workflow die digitalen und restaurativen Arbeitsabläufe Schritt für Schritt verfolgen. Besonders beliebt ist laut einer Anwenderin der iTero Intraoralscanner, da er selbst kariöse Läsionen erkennt und damit viel Zeit spart. Das physische Eventerlebnis wurde ergänzt durch die virtuelle Plattform, die daheimgebliebenen Messegästen Zugang zu den Seminarreihen und Tech-Talks bot. 


\section{Die IDS im Vergleich}

\begin{tabular}{lll}
\hline Fakten & 2021 & 2019 \\
\hline Aussteller & 830 aus 59 Ländern & 2327 aus 64 Ländern \\
\hline Fachbesucher & 23.000 aus 114 Ländern & 160.000 aus 166 Ländern \\
\hline $\begin{array}{l}\text { Ausstellungs- und Veranstal- } \\
\text { tungsfläche }\end{array}$ & $115.000 \mathrm{~m}^{2}$ & $177.000 \mathrm{~m}^{2}$ \\
\hline Auslandsanteil Aussteller & 72 Prozent & 73 Prozent \\
\hline Auslandsanteil Fachbesucher & 57 Prozent & 62 Prozent \\
\hline
\end{tabular}

\section{MEHR KOMFORT BEI DER FÜLLUNGSTHERAPIE}

Auch das Kerngeschäft der Zahnärzte, die Füllungstherapie, entwickelt sich kontinuierlich weiter und wird vor allem komfortabler. VOCO stellte mit VisCalor eine neue universelle Variante des thermoviskosen Komposits für alle Kavitätenklassen vor. Während das Bulk-Fill-Composite VisCalor bulk die einfache und schnelle Versorgung im Seitenzahnbereich ermöglicht, sind mit dem neuen VisCalor dank des großen Farbangebots an VITA-Farben auch sehr ästhetische Frontzahnrestaurationen möglich. Beide thermoviskosen Komposite sind nach Erwärmung erst fließfähig und lassen sich anschließend sofort modellieren.

Schütz Dental präsentierte Zahnarztpraxen wie Laboren mit Tizian 1.5 Pro ein kompaktes Frässystem mit erweiterten Anwendungsmöglichkeiten und extrem geringem Platzbedarf. Die leistungsfähige Hochfrequenzspindel ermöglicht Schnelligkeit und Präzision, das Schnellspannsystem einen komfortablen Wechsel von Haltersystemen bei gleichbleibender Präzision und der Bearbeitungswinkel die Erledigung selbst anspruchsvoller Aufgaben.

\section{INTEGRIERTE DIGITALE BEHANDLUNGSLÖSUNGEN}

Die Digitalisierung hat auch Einzug in die Behandlungseinheiten gehalten. Der dänische Hersteller XO CARE stellte seine mit dem Red Dot Design Award für innovative Produkte prämierte neue Einheit XO Flow vor. Dank integrierter digitaler Behandlungslösungen wird der Zahnarzt über ein Dashboard durch die Arbeitsschritte bei gleichzeitiger Einbindung von Standardprotokollen geführt. Die Behandlungen können mithilfe von Instrumenten-Voreinstellungen standardisiert werden.

\section{DIE ZUKUNFT IST DIGITAL}

Auch wenn die digitale Plattform IDSconnect in diesem Jahr aus der Not geboren wurde und Besucher wie Aussteller den persönlichen Kontakt vor Ort schätzen, zeichnet sich doch ab, dass das Hybrid-Format Zukunft hat.

Die durch die Corona-Pandemie beschleunigte Etablierung des digitalen Arbeitsplatzes inklusive Online-Meetings wird viele Geschäftsreisen und auch Messeteilnahmen künftig ersetzen. Ob sich dadurch auch für die nächste IDS eine Entschleunigung ergibt, bleibt abzuwarten. Wir werden es vom 14. bis 18. März 2023 erleben. 